\title{
The value of prophylactic (monthly) clotrimazole versus empiric self-treatment in recurrent vaginal candidiasis
}

\author{
I W Fong
}

\begin{abstract}
:
Objective-To determine the comparative efficacy and cost benefit of prophylactic monthly (perimenstrual) clotrimazole, versus empiric self-treatment with the same agent at the onset of symptoms in recurrent vulvovaginal candidiasis.

Design-Prospective, randomised, open cross-over study of women with proven recurrent vulvovaginal candidiasis. Clinical and microbiological assessments were done every two months for 12 months.

Setting-Women's Clinic of a University Teaching Hospital.

Subjects-Twenty-three otherwise healthy, non-pregnant women with greater than four proven episodes of candida vaginitis in the last year were enrolled into the study.

Intervention-Patients were randomised to receive: (1) a single dose of prophylactic clotrimazole $500 \mathrm{mg}$ ovule just before or on the last day of the menses each month for 6 months; (2) or a single dose of clotrimazole $500 \mathrm{mg}$ ovule empirically at the onset of symptoms for 6 months. After the first 6 months patients were crossed-over to the opposite regimen.

Main Outcome Measures-Symptoms of recurrent vulvovaginitis during each period, and number of clotrimazole ovules used for each 6 month period. The personal preference of the patients for the two different regimens were assessed at the end of study.
\end{abstract}

Results-During the prophylactic 6 months period of the study, 23 patients had 50 episodes of symptomatic vaginitis (mean $2 \cdot 2$ episodes per patient), versus 86 episodes (mean 3.7 episodes/patient) during the empiric self-treatment 6 months period $(P=0.05)$. However, during the prophylactic period a total of 168 clotrimazole ovules were used (mean $7 \cdot 3$ per patient), versus 84 ovules (mean 3.6 per patient) during the empiric selftreatment period, $p<0.001$.

The personal preference of the patients for the type of regimen employed were 17 $(73.9 \%)$ in favour of the empirical treatment, versus $4(17 \cdot 4 \%)$ in favour of the prophylactic treatment and $2(8.7 \%)$ no personal preference, $p<0.01$.

Conclusion-Empiric self-treatment is more cost-effective and preferable to patients than cyclical monthly prophy- lactic use of $500 \mathrm{mg}$ clotrimazole vaginal ovules.

(Genitourin Med 1994;70:124-126)

\section{Introduction}

Recurrent and persistent vulvo-vaginal candidiasis in otherwise healthy women is an uncommon condition that afflicts a small group of women, but most gynaecologists and family physicians have seen patients with this disease. The aetiology or reasons for recurrent infection are poorly understood ${ }^{12}$ and there is no known permanent cure. ${ }^{1}$

Chronic suppressive therapy using cyclical or daily continuous therapy with topical or oral antifungal agents ${ }^{34}$ have been used to control or suppress recurrences with success.

There is no study reported in the literature to date that compares a strategy of prophylactic cyclical antifungal agent to empiric selftreatment for symptomatic episodes. A study of this nature was done to compare the benefit of prophylactic perimenstrual clotrimazole ovules versus empiric self-treatment with the same agent, with respect to reduction of symptomatic episodes of vaginitis, cost-benefit and personal preference.

\section{Method}

A prospective, randomised, cross-over design study was conducted on 23 women with recurrent, clinical symptomatic vaginal candidiasis that was defined as greater than four episodes (microbiologically proven) in the past year. Informed consent was obtained from each patient and approval was obtained from the Clinical Trials Committee of the institution. Women with a consistent stable history of chronic recurrent candida vaginitis with no other chronic underlying illness (such as diabetes mellitus), no history of chronic use of antibiotics or steroids, were selected for the study. Patients were randomised to two regimens for 6 months periods:

(a) Clotrimazole $500 \mathrm{mg}$ vaginal ovules perimenstrually (once a month prophylactically), plus for symptomatic episodes in between. Patients with a history of recurrent symptoms before the menses were advised to insert the ovule 5-7 days before the expected menses. For patients with symptoms usually occurring after the menses, the ovule was inserted the last day of the period.

(b) Empiric self-treatment with single doses of clotrimazole $500 \mathrm{mg}$ ovules at onset \\ Medicine, St. \\ University of Toronto \\ Toronto, Canada.
}


of vaginitis symptoms.

After the first six months patients were crossed over to the opposite arm for another 6 months.

The random numbers were computer generated. Numbered, sealed envelopes containing the therapeutic regimen were kept in the Pharmacy Department, ensuring that the investigator remained blinded to the randomisation. Each patient was provided with a calendar diary to note the number of clotrimazole ovules for prophylactic and symptomatic uses for each month.

A clinical assessment was conducted initially and then every two months for one year. Vaginal washings (obtained by irrigation of 5 $\mathrm{ml}$ of sterile saline) and swabs from the vulva and vagina were cultured for yeast on Sabouraud's and blood agar. Identification of yeasts was done by the germ tube method using human serum, production of chlamydospores on Tween 90 and sugar assimilation when necessary. Initial genital swabs were cultured for Neisseria gonorrhoea, Gardnerella vaginalis, Chlamydia trachomatis and Trichomonas vaginalis.

\section{Statistical and Data Analysis:}

Comparison of the following parameters were made for the two regimens: (1) number of symptomatic episodes of vaginitis; (2) number of clotrimazole ovules used for each 6 months period; (3) personal preference of the patients for the different regimens. For continuous variables, the regimens were compared using the Student's $t$ test. For categorical variables, the groups were compared by chi square analysis or Fisher's exact test (if the number in any cell was less than five). Ninety-five percent confidence intervals (CI) were calculated for all results expressed as rates.

\section{Results}

Twenty-three patients were enrolled into the study. The mean age was 33 , SD $7 \cdot 4$ years (range 17-43 years) the mean number of vaginal candidiasis per year prior to enrolment was 7.5 episodes (range 5-12).

The overall results of the study is summarised in table I. During the prophylactic period of clotrimazole administration, patients experienced symptoms of vaginitis on 50 occasions ( $2 \cdot 2$ episodes per patient). On many occasions ( $42 \%$ of the time) the symptoms coincided with initiation of the prophylactic ovule before the menses and did not

Response to prophylactic and empiric use of clotrimazole $500 \mathrm{mg}$ ovules

\begin{tabular}{lccc}
\hline & $\begin{array}{l}\text { Prophylatic } \\
\text { clotrimazole } \\
N=23\end{array}$ & $\begin{array}{l}\text { Empiric } \\
\text { self-treatment } \\
N=23\end{array}$ & p value \\
\hline Vaginitis episodes & 50 & 86 & \\
Average episodes per patient & $2 \cdot 2 \pm 1.8$ & $3.1 \pm 2.9$ & 0.05 \\
Total ovules used & 168 & 84.67 .0 & $<0.001$ \\
(average per patient) & $7 \cdot 3 \pm 1.8$ & $3.6 \pm 3.0$ & $<0.001$ \\
Patient's preference & $4(17.4 \%)$ & $17(73.9 \%)$ & \\
CI & $2-32 \%$ & $56-92 \%$ & \\
\hline
\end{tabular}

$\mathrm{CI}=95 \%$ Confidence Interval. require extra treatment. On the other hand there were 86 episodes ( 3.8 episodes per patient) of symptomatic vaginitis during the empiric self-treatment, $\mathrm{p}=0.05$.

However, during the prophylactic period a total of 168 clotrimazole ovules were used (mean 7.3 ovules per patient), versus 84 ovules (mean 3.6 ovules per patient) during the empiric self-treatment period, $\mathrm{p}<0.001$. Moreover, on assessment of the personal preference of the patients for the two regimens or strategies used $17(73.9 \%)$ preferred the empiric self-treatment approach, 4 $(17 \cdot 4 \%)$ preferred the prophylactic approach and $2(8.7 \%)$ had no personal preference, $\mathrm{p}<0.05$.

The cost of the medications alone for the prophylactic 6 month-period was $\$ 2445.00$ (Canadian) or $\$ 106.50$ per patient, whereas the cost during the empiric self-treatment period was $\$ 1260.00$ or $\$ 54.80$ per patient.

\section{Discussion}

There is no known permanent cure for recurrent vulvovaginal candidiasis, and a variety of therapeutic modalities have been tried without success. ${ }^{1}$ Chronic suppressive (or prophylactic) therapy intermittently or continuously for several months has been used with success to control the patients' symptoms. However, after discontinuation of the prophylactic or suppressive therapy most patient will continue to have recurrences. ${ }^{3-5}$ Davidson and Mould $^{3}$ first showed that intermittent suppressive therapy with clotrimazole (cream and pessaries) on the 5th to 11 th day of the menstrual cycle was effective in reducing symptomatic disease. In this study, only a single clotrimazole $(500 \mathrm{mg}$ ) pessary was used, as this has been shown to be effective as a single dose therapy for acute Candida vaginitis. The timing of the prophylactic doses in this study depended on the pattern of recurrences of the previous vaginitis episodes and was, thus, individualised to the patient's needs.

In this study, patients with chronic recurrent vaginal candidiasis were used as their own controls, thus decreasing the interpatient variability. During 6 months of prophylactic therapy (138 patients months), there was a $30 \%$ reduction of symptomatic vaginitis episodes, and $42 \%$ of the symptoms during this period occurred at the time prophylaxis was being instituted (just before or after the menses) and did not require extra treatment. However, there was significantly less amount of clotrimazole ovules used during the empiric self-treatment period, compared with the prophylactic period, resulting in a $50 \%$ cost saving for the medication alone.

On assessing the patients' personal preference to the regimens used, the vast majority (74\%) preferred the empiric self-treatment strategy over the cyclical prophylactic method.

One of the limitations of this study is the small sample size (and some may argue, the cross-over design). However, the variability of 
data obtained with this design is less than that associated with the parallel design. Withinpatient differences are used to assess treatment differences in a cross-over trial. Because of its increased sensitivity and small variability, the cross over design requires fewer patients than does the parallel design to detect the same effect. ${ }^{6}$ The patients in this study had a chronic stable condition, and similar baseline condition was present at the start of two treatment periods. Moreover, neither form of treatment strategy was likely to have any residual or carryover effect. Also randomisation of the type of treatment period initiated should obviate any selection bias.

In summary, although perimenstrual clotrimazole prophylactically may decrease the number of symptomatic vaginitis episodes, empiric self-treatment was more cost effective and the preferred strategy by the majority of patients with chronic recurrent vaginal candidiasis. For patients with at least twelve episodes per year, the prophylactic strategy may be more beneficial.

1 Sobel JD. Vulvovaginal candidiasis-What we do and do not know. Ann Intern Med 1984;101:390-2.

2 Fong IW, McCleary P, Read S. Cellular immunity of patients with recurrent or refractory vulvovaginal moniliais. Am $\mathcal{F}$ Obstet Gynecol 1992;166:887-90.

3 Davidson B, Mould RF. Recurrent genital candidosis in women and effect of intermittent prophylactic treatment. Br $\mathcal{F}$ Veneral Dis 1978;54:176-83.

4 Sobel JD. Recurrent vulvovaginal candidiasis. A prospective study of the efficacy of maintenance ketoconazole therapy. $N$ Engl $¥$ Med 1986;1313:1455-8.

5 Fong IW. The value of chronic suppressive therapy with itraconazole versus clotrimazole in women with recurrent vaginal candidiasis. Genito-urin Med 1992;68 374-7.

6 Spilker B. Classification and Description of Phases I, II and III Clinical Trials Design. In: Guide to Clinical Trials. Ed. B. Spilker; 1991:27-43, New York, Raven Press. 